\title{
Smith-Purcell radiation from a charge moving above a grating of finite length and width
}

\author{
Amit S. Kesar* \\ Applied Physics Division, Soreq Nuclear Research Center, Yavne 81800, Israel
}

(Received 2 September 2009; published 23 February 2010)

\begin{abstract}
Smith-Purcell radiation (SPR), emitted when a charge passes above a periodic grating, is important for applications such as terahertz production and nondestructive bunch-length diagnostics. The grating width is shown to become an important parameter for accurately predicting the radiation, and especially in the highly relativistic regime where the charge wakefield considerably stretches in the transverse direction. The SPR radiation is rigorously calculated by the electric-field integral equation (EFIE) method for a grating of finite width and length. The integral equation is arranged as a multilevel block-Toeplitz matrix by using symmetry under translation with respect to the grating period and width directions. Following Barrowes et al. [Microw. Opt. Technol. Lett. 31, 28 (2001)] enhanced computational efficiency can be achieved by matrix to vector projection of the essential matrix elements. A numerical example is calculated for a relativistic $(\gamma=36), 1-\mathrm{mm}$ long, bunch traveling $0.6-\mathrm{mm}$ above a ten-period grating with a period of $2.0 \mathrm{~mm}$ and width of $10 \mathrm{~mm}$. The SPR resonance relationship and its broadening due to the finite number of grooves are consistent with the closed-form formulations. The surface current was shown to be concentrated along the center of the grating and decreasing towards its edges. The surface current, power spectrum, and radiated energy were compared to the EFIE formulation in which an infinitely wide grating was assumed. The above parameters resulted in considerable difference of up to a factor of 2.5 between the finite width and the infinitely wide grating assumption, which means that for accurate calculations the grating width should be taken into consideration. A general rule for the required grating width to achieve an accurate SPR radiation result relative to the infinite width result, and the expected accuracy by the infinite width assumption for most radiation angles, is provided.
\end{abstract}

DOI: 10.1103/PhysRevSTAB.13.022804

\section{INTRODUCTION}

The passing of a charge above a grating results in a broad radiation spectrum known as Smith-Purcell radiation (SPR) [1]. The $n$th order of the radiated wavelength $\lambda$ is correlated to the spatial observation angles $\theta$ and $\phi$ by the SPR resonance relationship:

$$
\lambda=\frac{D_{g}}{n}\left(\beta^{-1}-\sin \theta \sin \phi\right) .
$$

As illustrated in Fig. 1, the charge is passing at a relativistic velocity $\beta=v_{x} / c=\left(1-\gamma^{-2}\right)^{1 / 2}$ above a grating of periodic length $D_{g}$ in which the grooves are parallel to the $y$ axis. The components of the wave number are $k_{x}=$ $k \sin \phi \sin \theta, k_{y}=k \cos \phi$, and $k_{z}=k \sin \phi \cos \theta$, where $k=\omega / c$, and $\omega$ is the angular frequency.

SPR can be described by the charge wake diffracting upon the grating grooves [2]. Therefore, the charge will lose some of its energy by this mechanism. The common formulation for SPR is used when the lost energy is negligible compared to its initial energy. This happens, typically, when the charge is traveling at relativistic velocities.

The evanescent waves from the grating can be used to bunch an electron beam. This bunching occurs at a frequency which is below the SPR spectrum. However, the

\footnotetext{
*kesar@soreq.gov.il
}

PACS numbers: 29.27.-a, 02.70.Dh, 42.25.Fx, 42.79.Dj

higher harmonics of the bunching frequency will emit at angles according to the SPR resonance relationship (1). This mechanism is known as Smith-Purcell free-electron laser (SP-FEL) [3]. Absolute and convective instabilities of SPR interaction were studied in [4].

SPR can be used as a coherent terahertz radiation source. Li, Sun, and Kim [5] proposed using a photoemission rf gun at an energy of a few $\mathrm{MeV}$ and beam current of $1 \mathrm{~mA}$ to produce hundreds of watts of $\mathrm{THz}$ radiation. They also

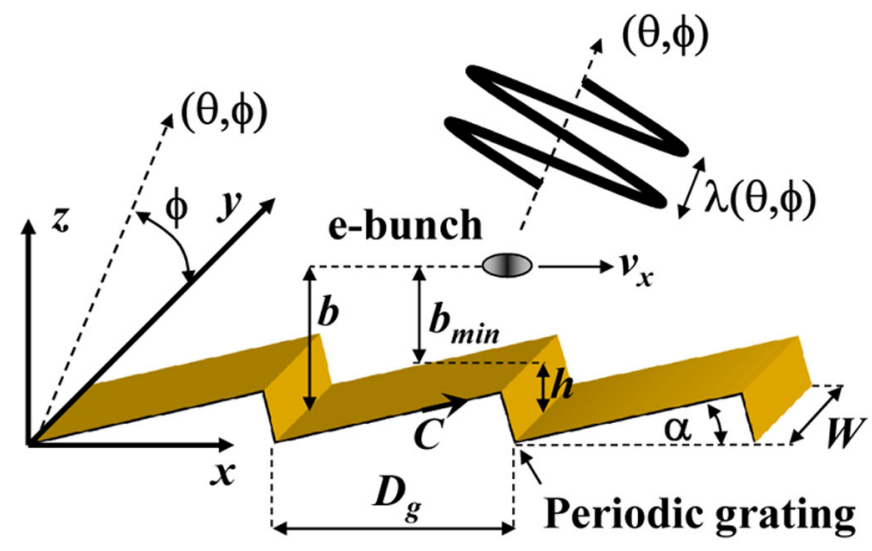

FIG. 1. (Color) The SPR scheme. An electron bunch is traveling at an axial velocity $v_{x}$ above an echelle grating of finite dimensions. 
predict that even higher radiated power can be generated by extending the grating to obtain a SP-FEL interaction. Submillimeter wavelengths were observed by Andrews et al. in a SP-FEL setup with a $\sim 30 \mathrm{keV}$ beam [6]. SPR in a comb of narrow band frequencies extending up to the $\mathrm{THz}$ region was obtained by a train of relativistic electrons from the 17-GHz MIT linac [7]. The theory for emission of light by unconventional SPR mechanism using ultrarelativistic electrons traveling near a finite-size photonic crystal has been reported by Ochiai and Ohtaka [8].

SPR can also be used as a nondestructive bunch-length measurement tool [9-11]. The longitudinal bunch profile can be estimated by a fitting method [12-14]. A recent advance in bunch profile measurement by SPR experiment was obtained by using the Kramers-Kronig dispersion relations and assuming that the profile is of finite duration [15]. This experiment was conducted in the highly relativistic regime with a $28.5 \mathrm{GeV}$ beam.

Numerous methods for calculating the SPR energy were published. Assuming infinite periodic boundary conditions, rigorous models from a line [16] and a point [17] charge were developed by van den Berg, and were later calculated for relativistic energies by Haeberlé et al. [18]. Fair agreement with van den Berg's model was obtained by Kube et al. for optical SPR by a beam energy of $855 \mathrm{MeV}$. Using the modal expansion method, calculations of SPR from a volume strip grating are presented by Kube in [19].

A surface current model based on the image-charge approximation and its optimization for very high energies are described in [20,21]. While 6 orders of magnitude difference were obtained by comparing this model to the results in [22], the experiments described in $[12,13,15,23]$ demonstrated reasonable agreement with this model. A comparison between van den Berg's model, the surface current model, and the resonant diffraction radiation model for volume and thin strip gratings is presented by Karlovets and Potylitsyn in [24].

SPR calculations by 2D and 3D particle-in-cell (PIC) simulation codes are presented in [25-27]. Both SPR and SP-FEL interactions were simulated by this method. However, due to the finite computation size, the radiation was measured in the near field. PIC studies show that the start current in a Smith-Purcell backward wave oscillator can be reduced by using a sidewall grating [28].

A model based on the finite-difference time-domain (FDTD) method, in which a total-field scattered-field technique combined with a near- to far-field projection, is described in [29]. A frequency-domain model based on the electric-field integral equation (EFIE) extends van den Berg's model for practical gratings of finite length and agrees with the FDTD model [29,30]. Using roomtemperature diode detectors, accurate far-field measurements of SPR from a $15 \mathrm{MeV}$ train of bunches support the validity of this model.

In many of the papers cited above, the validity of the various SPR models for a wide range of beam energies has been addressed. As was shown in [29,30], due to the relativistic effect it is important to take into account the number of grating periods in practical SPR setups. Furthermore, at highly relativistic energies the bunch wake in the transverse direction becomes considerably wide. In such cases models that assume an infinitely wide grating may become inaccurate. The objectives of this paper are to demonstrate the conditions in which the grating width has to be taken into account and to extend the EFIE model in [30] for this case. This model may be important for terahertz production by SPR interaction and for nondestructive bunch-length measurements by SPR.

\section{EFIE FORMULATION}

A charge $q$ with a spatial distribution $f\left(x_{0}, y_{0}, z_{0}\right)$ moving in free space along the $x$ direction at a relativistic velocity $\beta$ has an electromagnetic (EM) wake. Assuming a frequency dependence $e^{j \omega t}$, the Fourier transform of the wake's electric field incident on the grating is given by

$$
\begin{aligned}
\mathbf{E}^{\text {inc }}(\mathbf{r}, \omega)= & \frac{q Z_{0} k}{2 \pi \beta^{2} \gamma} \iiint e^{-j(k / \beta)\left(x-x_{0}\right)} \\
& \times\left[\frac{\hat{y}\left(y-y_{0}\right)+\hat{z}\left(z-z_{0}\right)}{\rho} K_{1}\left(\frac{k \rho}{\beta \gamma}\right)\right. \\
& \left.+j \frac{\hat{x}}{\gamma} K_{0}\left(\frac{k \rho}{\beta \gamma}\right)\right] f\left(x_{0}, y_{0}, z_{0}\right) d x_{0} d y_{0} d z_{0},
\end{aligned}
$$

where $\rho=\left[\left(y-y_{0}\right)^{2}+\left(z-z_{0}\right)^{2}\right]^{1 / 2}$ is the transverse distance, and $K_{0}$ and $K_{1}$ are the zeroth and first order modified Bessel functions of the second kind, respectively. The freespace impedance is $Z_{0}=\left(\mu_{0} / \epsilon_{0}\right)^{1 / 2}$, where the free-space permeability and permittivity are $\mu_{0}$ and $\epsilon_{0}$, respectively.

Throughout this paper it is assumed that the charge spatial distribution is a Gaussian function with a full width at half maximum (FWHM) of $\sigma_{x}$ in the longitudinal direction, and a $\delta$ function at $y_{0}=0$ and $z_{0}=b$ in the transverse direction. The coupling of the charge to the grating is affected by $b$ as shown in Fig. 1 , where $b_{\min }=$ $b-h$ is a positive number and $h$ is the grating height. For gratings of finite width, and especially at relativistic energies, the coupling of the charge to the grating may not decay exponentially but rather as the modified Bessel functions as will be shown below.

As the charge passes above a metallic grating it induces a surface current $\mathbf{J}(\mathbf{r}, \omega)$ on the perimeter of the grating. At each angular frequency $\omega$ the vector potential of the reflected field $\mathbf{A}(\mathbf{r}, \omega)$ caused by a surface current is given by $[31,32]$

$$
\mathbf{A}(\mathbf{r}, \omega)=\int_{-W / 2}^{W / 2} \int_{C} \mathbf{J}\left(\mathbf{r}^{\prime}, \omega\right) G\left(\mathbf{r}-\mathbf{r}^{\prime}, \omega\right) d c^{\prime} d y^{\prime},
$$

where $\mathbf{r}=x \hat{x}+y \hat{y}+z \hat{z}$ and $\mathbf{r}^{\prime}=x^{\prime} \hat{x}+y^{\prime} \hat{y}+z^{\prime} \hat{z}$ are the observation and source points, respectively, and the freespace Green's function which correlates the radiation from 
the source to the observation point is

$$
G\left(\mathbf{r}-\mathbf{r}^{\prime}, \omega\right)=\frac{e^{-j k\left|\mathbf{r}-\mathbf{r}^{\prime}\right|}}{4 \pi\left|\mathbf{r}-\mathbf{r}^{\prime}\right|}
$$

The reflected electric field can be derived from the vector potential by

$$
\mathbf{E}^{\mathrm{ref}}(\mathbf{r}, \omega)=-j \frac{Z_{0}}{k}\left(\nabla \nabla+k^{2}\right) \mathbf{A}(\mathbf{r}, \omega) .
$$

In the case of a perfectly conducting grating the tangential component of the electric field on the grating surface is zero, i.e. $\mathbf{E}_{\|}=\mathbf{E}^{\text {inc }}+\mathbf{E}^{\text {ref }}=0$. In order to find the unknown surface current, Eqs. (2) and (3) can be combined into two coupled electric-field integral equations solved along the grating perimeter, namely, the source and observation points are located on the grating surface,

$$
\begin{aligned}
E_{c}^{\mathrm{inc}}(\mathbf{r}, \omega)= & j \frac{Z_{0}}{k} \int_{-W / 2}^{W / 2} \int_{C}\left[J_{c} \mathcal{A} G\left(\mathbf{r}-\mathbf{r}^{\prime}, \omega\right)\right. \\
& \left.+J_{y} \mathcal{B} G\left(\mathbf{r}-\mathbf{r}^{\prime}, \omega\right)\right] d c^{\prime} d y^{\prime} \\
E_{y}^{\mathrm{inc}}(\mathbf{r}, \omega)= & j \frac{Z_{0}}{k} \int_{-W / 2}^{W / 2} \int_{C}\left[J_{c} \mathcal{C} G\left(\mathbf{r}-\mathbf{r}^{\prime}, \omega\right)\right. \\
& \left.+J_{y} \mathcal{D} G\left(\mathbf{r}-\mathbf{r}^{\prime}, \omega\right)\right] d c^{\prime} d y^{\prime} .
\end{aligned}
$$

The spatial electric-field components tangent to the grating profile and along the grating grooves are $E_{c}^{\text {inc }}(\mathbf{r}, \omega)=E_{x}^{\text {inc }}(\mathbf{r}, \omega) \cos \alpha+E_{z}^{\text {inc }}(\mathbf{r}, \omega) \sin \alpha \quad$ and $E_{y}^{\text {inc }}(\mathbf{r}, \omega)$, respectively. The corresponding spatial components of the surface current are $J_{c}\left(\mathbf{r}^{\prime}, \omega\right)=J_{x}\left(\mathbf{r}^{\prime}, \omega\right) \times$ $\cos \alpha^{\prime}+J_{z}\left(\mathbf{r}^{\prime}, \omega\right) \sin \alpha^{\prime}$ and $J_{y}\left(\mathbf{r}^{\prime}, \omega\right)$, respectively, where $\alpha$ and $\alpha^{\prime}$ are the observation and source angles tangent to the grating profile, respectively. The operators acting on the Green's function are

$$
\begin{aligned}
\mathcal{A}= & k^{2} \cos \left(\alpha-\alpha^{\prime}\right)+\cos \alpha \cos \alpha^{\prime} \partial_{x}^{2}+\sin \alpha \sin \alpha^{\prime} \partial_{z}^{2} \\
& +\sin \left(\alpha+\alpha^{\prime}\right) \partial_{x z} \\
\mathcal{B}= & \cos \alpha \partial_{x y}+\sin \alpha \partial_{z y} \\
\mathcal{C}= & \cos \alpha^{\prime} \partial_{x y}+\sin \alpha^{\prime} \partial_{z y} \\
\mathcal{D}= & k^{2}+\partial_{y}^{2}
\end{aligned}
$$

The unknown surface currents in Eqs. (6a) and (6b) were solved by dividing the grating surface into $N_{c}$ straight segments along its profile and $N_{y}$ straight segments along its width. Therefore, the grating was divided into $N=$ $N_{c} N_{y}$ straight surface segments, each of them is of area $\Delta_{n}$, and it was assumed a piecewise constant surface current in each one,

$$
\begin{aligned}
& J_{c}(c, y, \omega) \approx \sum_{n=1}^{N} a_{n}(\omega) g_{n}(c, y) \\
& J_{y}(c, y, \omega) \approx \sum_{n=1}^{N} b_{n}(\omega) g_{n}(c, y),
\end{aligned}
$$

where $g_{n}(c, y)=1$ at the $n$th segment and zero out of it, and $a_{n}(\omega)$ and $b_{n}(\omega)$ are the unknown variables constructing the piecewise constant surface current components. Thus, Eqs. (6a) and (6b) were approximated by a set of $2 N$ linear equations:

$$
\left[\begin{array}{l}
E_{c m} \\
E_{y m}
\end{array}\right]=\left[\begin{array}{ll}
\mathcal{A}_{m n} & \mathcal{B}_{m n} \\
\mathcal{C}_{m n} & \mathcal{D}_{m n}
\end{array}\right]\left[\begin{array}{l}
a_{n} \\
b_{n}
\end{array}\right],
$$

where $E_{c m}$ and $E_{y m}$ are the incident electric components given by Eq. (2) at the center of the $m$ th observation surface segment. The matrix terms were calculated numerically, where its diagonal terms which are related to the Green's function singularity were calculated by placing the observation point at a small distance above the grating surface and by approaching this distance to zero.

The above division of the grating into rectangular segments along its profile and width results in a symmetry under translation along the grating grooves and along the width directions. Therefore the matrix in Eq. (9) can be arranged as a four-level block matrix in which its first two levels are block-Toeplitz matrices. For example, the first level uses the symmetry under translation along the grating width, in which there are $N_{y}$ elements,

$$
\mathbf{A}=\left[\begin{array}{cccc}
\mathbf{a}_{0}^{1} & \mathbf{a}_{1}^{1} & \ldots & \mathbf{a}_{N_{y}-1}^{1} \\
\mathbf{a}_{-1}^{1} & \mathbf{a}_{0}^{1} & & \\
\vdots & & \ddots & \vdots \\
\mathbf{a}_{-N_{y}+1}^{1} & & \ldots & \mathbf{a}_{0}^{1}
\end{array}\right]
$$

The second level uses the symmetry under translation along the grating periods. For a grating with $N_{g}$ grooves, the block-Toeplitz matrix of this level will have the form

$$
\mathbf{a}_{-N_{y}+1}^{1}, \ldots, \mathbf{a}_{N_{y}-1}^{1}=\left[\begin{array}{cccc}
\mathbf{a}_{0}^{2} & \mathbf{a}_{1}^{2} & \ldots & \mathbf{a}_{N_{g}-1}^{2} \\
\mathbf{a}_{-1}^{2} & \mathbf{a}_{0}^{2} & & \\
\vdots & & \ddots & \vdots \\
\mathbf{a}_{-N_{g}+1}^{2} & & \ldots & \mathbf{a}_{0}^{2}
\end{array}\right] .
$$

The third level is made out of the elements which represent the interconnections between the samples along each of the grating grooves, $N_{i}=N_{c} / N_{g}$. The last level is a $2 \times 2$ matrix which is made of the $\mathcal{A}, \mathcal{B}, \mathcal{C}$, and $\mathcal{D}$ elements in Eq. (7) which correlate $a_{n}$ and $b_{n}$ to $E_{c m}$ and $E_{y m}$ as shown in Eq. (9).

The above partitioning allows an increase in the numerical efficiency when solving Eq. (9) by following the algorithm described in Ref. [33]. In this paper the multilevel 
block-Toeplitz matrix is transformed into a vector that contains only the essential elements and has a size of $\left(2 N_{y}-1\right)\left(2 N_{g}-1\right)\left(2 N_{i}\right)^{2}$. The solution vector is padded with zeros at appropriate places and the result is a convolution of the two vectors. The solution vector, i.e., the unknown coefficients of the surface current components, was solved by the conjugate gradient algorithm and by taking zero as its initial guess [32].

The vector potential in the far field, $\mathbf{r} \gg \mathbf{r}^{\prime}$ is approximated as

$$
\begin{aligned}
\mathbf{A}^{\operatorname{far}}(r, \theta, \phi, \omega) \simeq & \frac{e^{-j k r}}{4 \pi r} \int_{-W / 2}^{W / 2}\left[\int_{C} \mathbf{J}\left(\mathbf{r}^{\prime}, \omega\right) e^{j k_{x} x^{\prime}+j k_{z} z^{\prime}} d c^{\prime}\right] \\
& \times e^{j k_{y} y^{\prime}} d y^{\prime},
\end{aligned}
$$

where $\mathbf{J}\left(\mathbf{r}^{\prime}, \omega\right)=\hat{x} J_{c} \cos \alpha^{\prime}+\hat{y} J_{y}+\hat{z} J_{c} \sin \alpha^{\prime}$. The magnetic component of the far field is approximated by

$$
\mathbf{H}(r, \theta, \phi, \omega) \simeq-j \mathbf{k} \times \mathbf{A}^{\mathrm{far}},
$$

and the power spectrum is

$$
P_{s}(\theta, \phi, \omega)=Z_{0} r^{2}|\mathbf{H}(r, \theta, \phi, \omega)|^{2} .
$$

Because of the finite number of grating grooves, $N_{g}$, the spectral content at each observation angle $(\theta, \phi)$ is broadened around the SPR resonance relationship in Eq. (1) and has a relative bandwidth of $1 / n N_{g}$. The angular distribution of the $n$th order of radiated energy is given by Parseval's theorem,

$$
E_{n}(\theta, \phi)=\frac{1}{\pi} \int_{\omega_{1}(n-0.5)}^{\omega_{1}(n+0.5)} P_{s}(\theta, \phi, \omega) d \omega,
$$

where the angular frequency of the first order is $\omega_{1}=$ $2 \pi c / D_{g}\left(\beta^{-1}-\sin \theta \sin \phi\right)$. For coherent radiation it is sufficient to calculate up to a maximum cutoff frequency of $\omega=2 \pi c / \sigma_{x}$.

\section{NUMERICAL EXAMPLE}

A numerical example of SPR from an echelle grating of finite width and length is presented in the following section. The bunch and grating parameters are as listed in Table I, unless specified otherwise.

TABLE I. Smith-Purcell radiation parameters.

Bunch charge, $q$

Bunch relativistic factor, $\gamma$

Height above the grating, $b_{\min }$

Bunch length, $\sigma_{x}$

Grating width, $W$

Grating period, $D_{g}$

Blaze angle, $\alpha$

Number of grooves, $N_{g}$

EFIE resolution, $\Delta_{n}$
In order to preestimate the conditions in which the grating width has to be taken into account, the contribution of the incident electric-field components of Eq. (2) is analyzed as follows. At relativistic velocities the axial component (in the $x$ direction) is negligible with respect to the other components since it scales as $1 / \gamma$. Assuming the bunch is centered with respect to the $y$ direction above the grating, the effective width of the component parallel to the grating grooves, $W_{y}$, and of the component transverse to the grating plane, $W_{z}$, are defined in this example as the widths in which the integration is $90 \%$ from that of an infinitely wide grating,

$$
\begin{gathered}
\frac{\int_{-W_{y} / 2}^{W_{y} / 2}(y / \rho) K_{1}(k \rho / \beta \gamma) d y}{\int_{-\infty}^{\infty}(y / \rho) K_{1}(k \rho / \beta \gamma) d y}=0.9 \\
\frac{\int_{-W_{z} / 2}^{W_{z} / 2}\left(b_{\min } / \rho\right) K_{1}(k \rho / \beta \gamma) d y}{\int_{-\infty}^{\infty}\left(b_{\min } / \rho\right) K_{1}(k \rho / \beta \gamma) d y}=0.9 .
\end{gathered}
$$

The effective width of these components vs frequency, calculated for $\gamma=36$ [30] and $\gamma=4.52$ [13], is shown in Fig. 2. As seen in this example $W_{y}$ is the larger component which suggests a wider grating. The higher relativistic factor $(\gamma=36)$ suggests a wider grating since the bunch wake stretches longer in the transverse direction. In this example, for $\gamma=36$ and $b_{\min }=0.6 \mathrm{~mm}$, the grating width should be taken into account if its width is less than $70 \mathrm{~mm}$.

The real and imaginary parts of the surface current components $J_{c}$ and $J_{y}$ vs the grating width and length, calculated for a 10-mm wide grating at a Fourier frequency of $150 \mathrm{GHz}$, are shown in Fig. 3. These currents were normalized to a point charge of $q=1 \mathrm{C}$. Since the charge travels above the center of the grating $J_{c}$ is an even

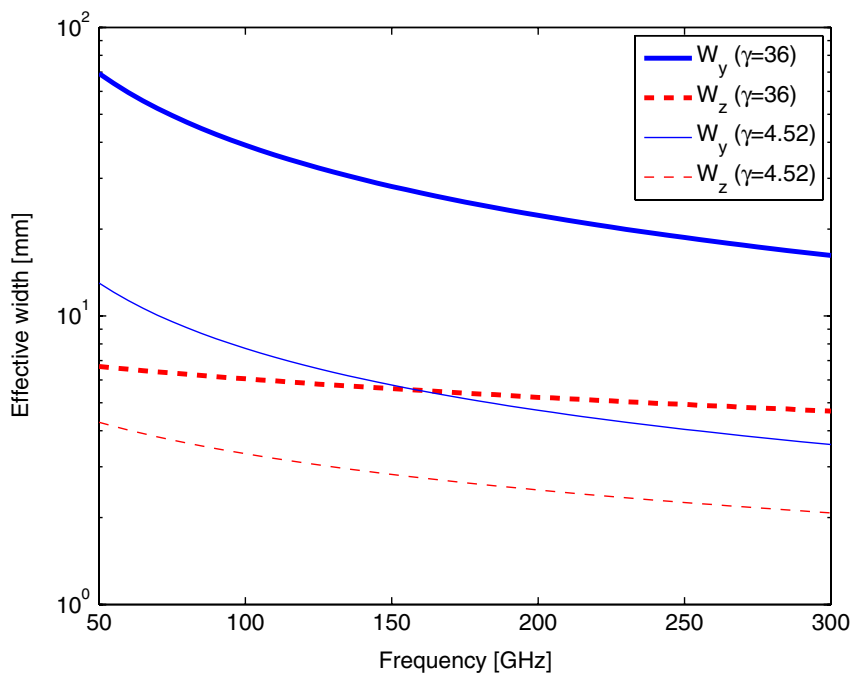

FIG. 2. (Color) Effective width of the parallel (solid lines) and transverse (dashed lines) components vs frequency for a charge traveling $0.6 \mathrm{~mm}$ above a grating. The effective width is calculated for $\gamma=36$ (thick lines) and $\gamma=4.52$ (thin lines). 

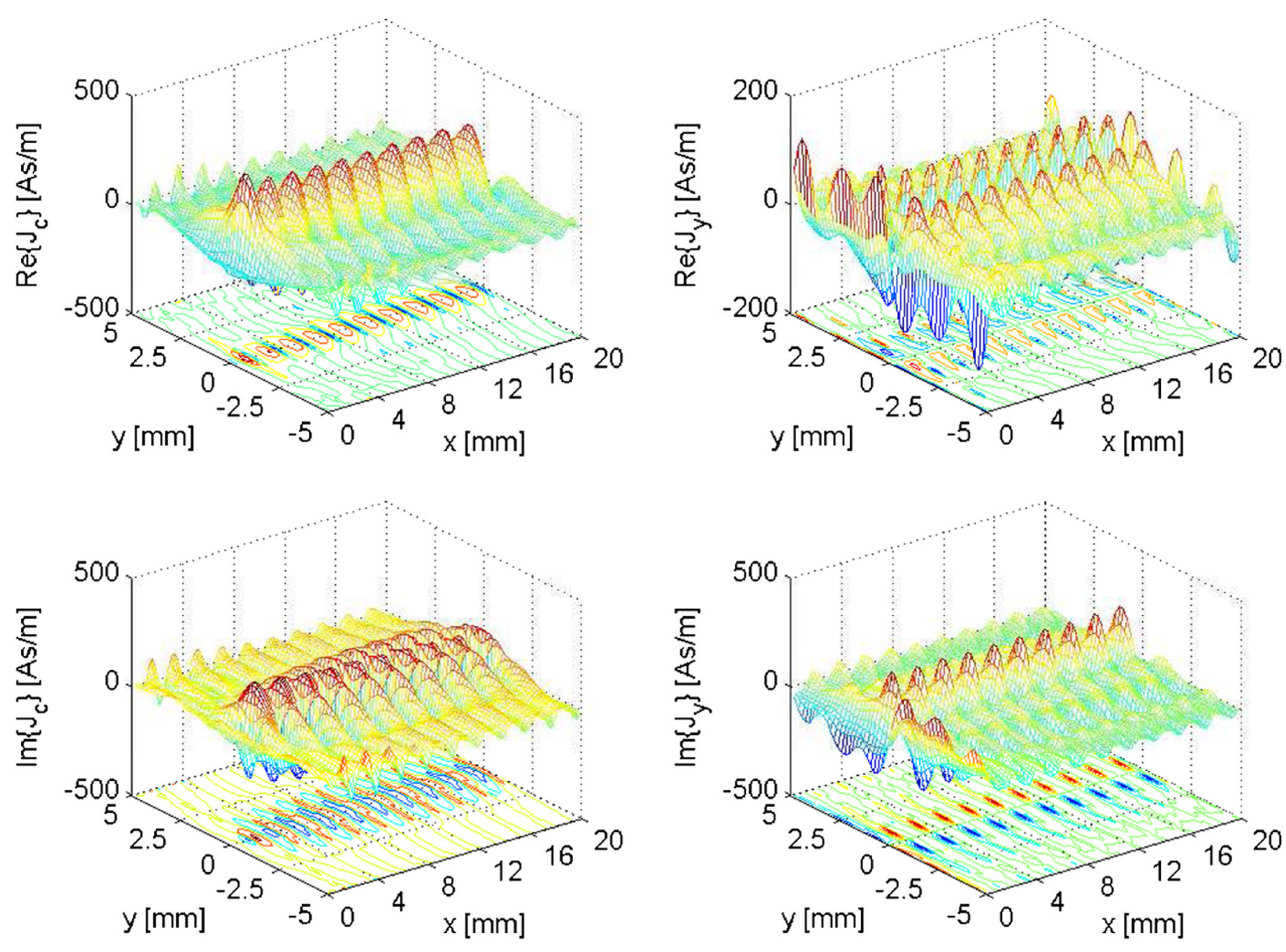

FIG. 3. (Color) Real and imaginary parts of the surface current components $J_{c}$ and $J_{y}$ vs the grating width and length at a Fourier frequency of $150 \mathrm{GHz}$.

function with respect to the grating width, $J_{c}(y)=J_{c}(-y)$, whereas $J_{y}$ is an odd function, $J_{y}(y)=-J_{y}(-y)$. As shown in this figure the currents are concentrated in the middle $5 \mathrm{~mm}$ of the grating and decay towards its edges. Similar surface currents were obtained for a highly relativistic case of $\gamma=10^{4}$. For a case of $b_{\min }=1 \mathrm{~mm}$ the surface currents were spread wider in the $y$ direction, concentrated in the middle $6 \mathrm{~mm}$ of the grating and decaying towards its edges.

As an example, it is possible to compose the real surface current density by the inverse transform of all of the Fourier components, $\mathbf{J}(\mathbf{r}, t)=(2 \pi)^{-1} \int \mathbf{J}(\mathbf{r}, \omega) e^{j \omega t}$. Figure 4 shows plots of the longitudinal current density component $J_{c}$ vs the grating width and length when the $50 \mathrm{pC}, \gamma=36,1 \mathrm{~mm}$ long bunch is located at $x=$ $0.75 \mathrm{~mm}$ and $y=0 \mathrm{~mm}$ (a), and at $x=11 \mathrm{~mm}$ and $y=$ $0 \mathrm{~mm}$ (b). In this figure it is possible to see the footprint of the bunch on the grating. This footprint is concentrated below the bunch in an area roughly $2 \mathrm{~mm}$ wide and $1 \mathrm{~mm}$ long in Fig. 4(a) and $2 \mathrm{~mm}$ long in Fig. 4(b).
It is interesting to compare the surface currents to those calculated for an infinitely wide grating [30]. This can be done by projection of the surface currents for each $\phi$ angle,

$$
\tilde{\mathbf{J}}\left(c, k_{y}, \omega\right)=\int_{-W / 2}^{W / 2} \mathbf{J}(c, y, \omega) e^{j k_{y} y} d y,
$$

where $k_{y}=k \cos \phi$. An example of this comparison is shown in Fig. 5 where the surface currents in Fig. 3 were projected by Eq. (17) for $\phi=70^{\circ}$. The real and imaginary parts of $J_{c}$ and $J_{y}$ by the finite-width EFIE model are shown by the solid lines. The surface currents by the infinitely wide grating assumption [30] are shown by the dashed lines. The resulted power spectrum was $27 \%$ lower for the finite-width grating as compared to the infinitely wide grating. For $\phi$ angles of 90, 80, 60, 50, and 40 degrees the power spectrum was $60 \%, 12 \%, 2 \%, 23 \%$, and $27 \%$ lower, whereas for 30 degrees it was $42 \%$ higher.

The first $(n=1)$, second $(n=2)$, and third $(n=3)$ orders of the SPR energy by the ten-period grating are shown in Figs. 6(a)-6(c), respectively, as contours in $\mathrm{nJ} / \mathrm{sr}$ 

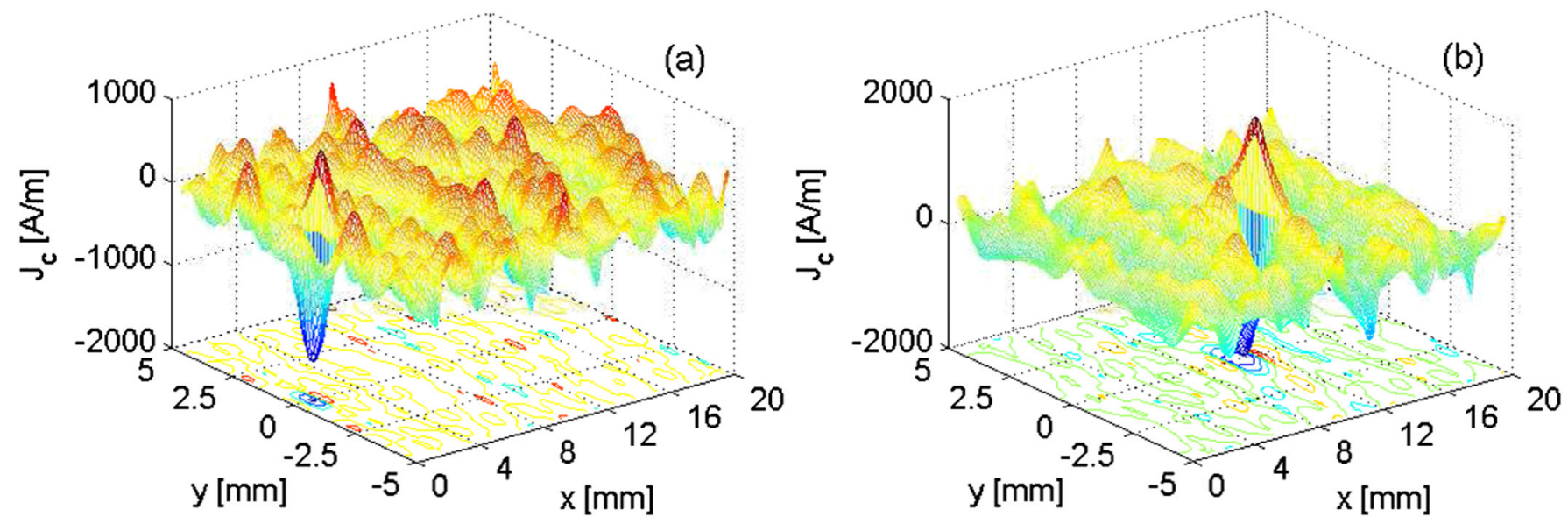

FIG. 4. (Color) Surface current $J_{c}$ vs the grating width and length when the $50 \mathrm{pC}$ bunch is at $y=0$ and $x=0.75 \mathrm{~mm}$ (a), and $x=11 \mathrm{~mm}(\mathrm{~b})$.
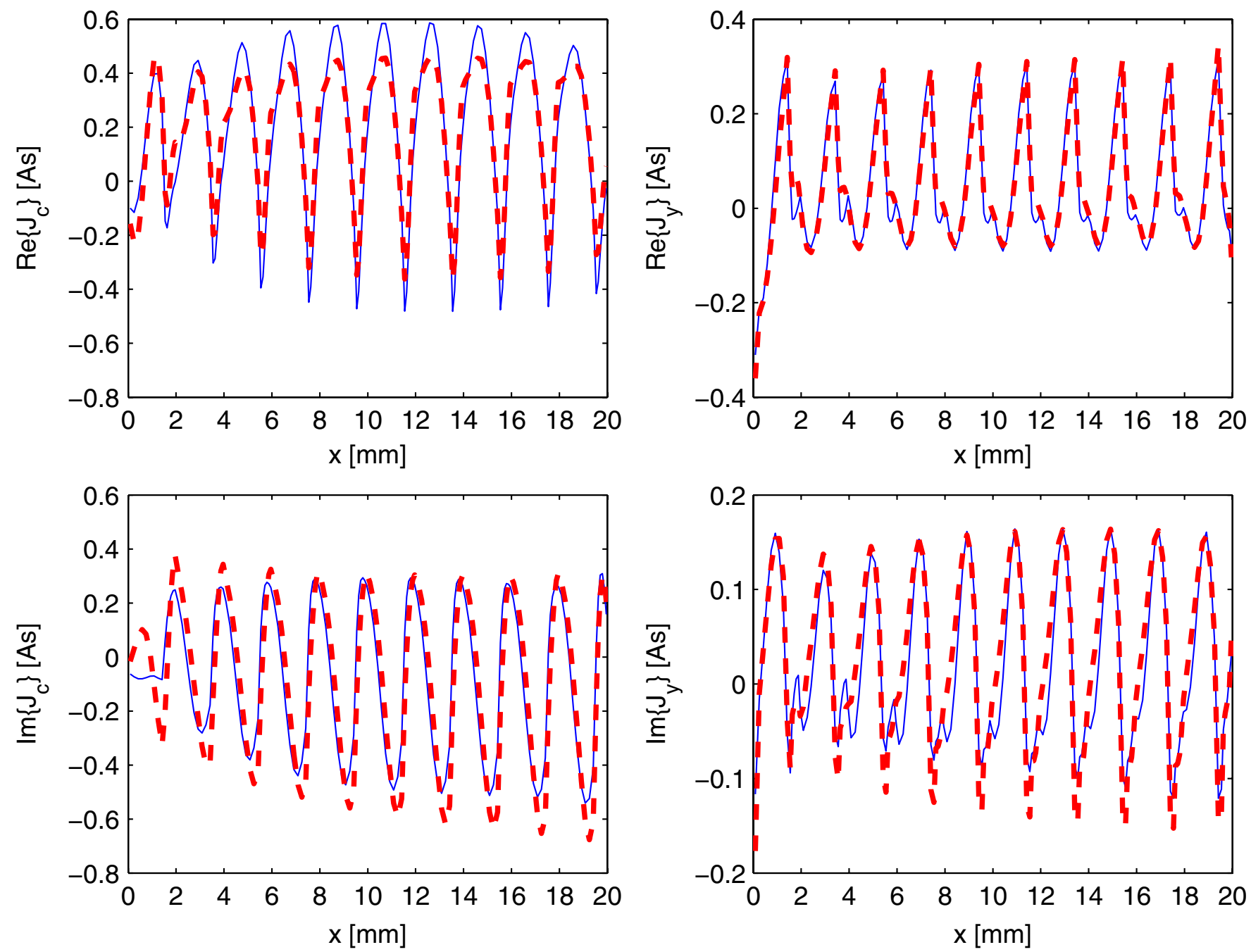

FIG. 5. (Color) Projection by Eq. (17) of the surface currents shown in Fig. 3 for $\phi=70^{\circ}$ vs the grating length (solid lines). These currents are compared to the case of an infinitely wide grating [30] (dashed lines). 

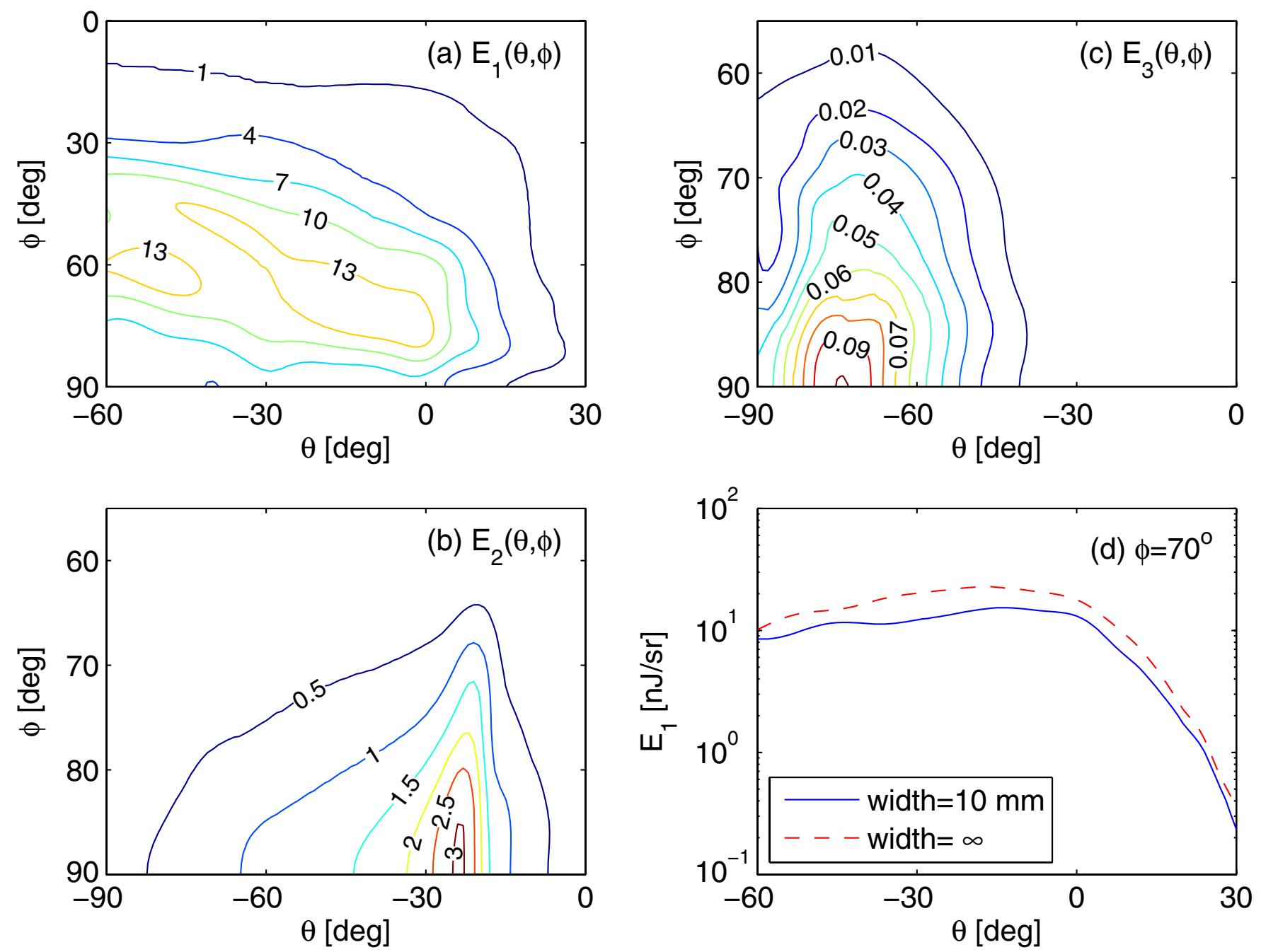

FIG. 6. (Color) Contour plot of the radiated energy in $\mathrm{nJ} / \mathrm{sr}$ vs observation angles $\theta$ and $\phi$ for the first (a), second (b), and third (c) Smith-Purcell orders calculated by Eq. (15). (d) First order radiated energy for $\phi=70^{\circ}$ by the 10-mm wide grating (solid line) compared to the radiated energy by an infinitely wide grating [30] (dashed line).

vs observation angles $\theta$ and $\phi$. The calculation is symmetric around $\phi=90^{\circ}$. As seen in this figure the energy for higher SPR orders drops and shifts to lower $\theta$ angles.

The first order radiated energy for $\phi=70^{\circ}$ is shown in Fig. 6(d) by the $10-\mathrm{mm}$ wide grating (solid line). This radiation is compared to the case of an infinitely wide grating [30] (dashed line). As seen in this figure the 10$\mathrm{mm}$ wide grating resulted in $\sim 20 \%-30 \%$ lower radiation compared to the infinitely wide grating, in agreement with the power spectrum results obtained from Fig. 5.

\section{DISCUSSION}

The grating width was shown to be an important parameter for calculating SPR radiation and especially for a highly relativistic charge in which its wake stretches in the transverse direction. The EFIE model in [30] for a bunch moving above a grating of finite length was extended to the case of a finite length and width. Using symmetry under translation for the grating setup and its division to rectangular segments allowed using the efficient algorithm described in [33]. This model, and the use of symmetry rules, can be applied to other radiation calculations such as transition and diffraction radiation.

The surface current in Fig. 3 was strong at the center 5$\mathrm{mm}$ of the grating with respect to the $y$ axis, both at the relativistic $(\gamma=36)$ and the highly relativistic $\left(\gamma=10^{4}\right)$ cases. This is probably because the induced surface current is mostly enhanced by spatial derivatives of the bunch wakefield. The spreading of the surface current in the $y$ direction as the bunch travels higher above the grating is in agreement with the theoretical expectation. A slight edge effect is seen in Figs. 3 and 4, where the current density has some increase towards the grating edges, $y= \pm 5 \mathrm{~mm}$. This effect is probably related to the wide transverse wakefield of the finite charge.

The $1 \mathrm{~mm}$ longitudinal footprint in Fig. 4(a) is in agreement with the bunch FWHM. When the bunch continues to 
propagate above the grating it induces a similar footprint below it. However, due to the finite-length effect, this footprint will be mixed with surface fields (and currents) in the $x$ direction from previous grating grooves [30], resulting in a change of the total longitudinal footprint.

The surface current and the resulting power spectrum were compared to those obtained from an infinitely wide grating assumption by projection to several $\phi$ angles at the Fourier frequency of $150 \mathrm{GHz}$. Considerable differences were found. At $\phi=90^{\circ}$ the finite-width grating resulted in a power spectrum 2.5 times lower compared to the infinitely wide grating assumption whereas at $\phi=30^{\circ}$ it was 1.4 times higher. For $\phi=70^{\circ}$ the radiated energy vs $\theta$ was $\sim 20 \%-30 \%$ lower. Therefore, in order to obtain accurate results it is important to consider by this model whether the grating width should be taken into account. Based on the numerical examples and the analysis that followed, a general rule for the required grating width to achieve an accurate SPR radiation result relative to the infinite width result would be $\sim 3-5$ times wider than $W_{z}$ defined in Eq. (16b). The estimated accuracy would be $>70 \%$ for most radiation angles.

The SPR model which includes a grating with finite dimensions is important for studying terahertz generation since the surface current and, consequently, the EM fields at the location of the beam current can be calculated directly in order to determine the coupling factor and the device gain. The model is also important for nondestructive bunch-length diagnostics, and especially for highly relativistic charges. It will be of high interest to compare these full 3D results to other models such as the surface current model [34].

\section{ACKNOWLEDGMENTS}

The author would like to thank Richard J. Temkin at the Massachusetts Institute of Technology (U.S.A.) and George Doucas at the University of Oxford (U.K.) for their important comments on this manuscript. The technical assistance of Assaf Hazzan, Lev M. Merensky, Ron Sapunar, Avi Shahadi, Tamir Siman-Tov, and Ady Wiernik (Israel) is gratefully acknowledged.

[1] S. J. Smith and E. M. Purcell, Phys. Rev. 92, 1069 (1953).

[2] G. Toraldo Di Francia, Nuovo Cimento 16, 61 (1960).

[3] L. Schächter and A. Ron, Phys. Rev. A 40, 876 (1989).

[4] O. Watanabe, T. Watanabe, K. Ogura, Y. Tatematsu, Y. Shima, and T. Imai, Jpn. J. Appl. Phys. 46, 6051 (2007).

[5] Y. Li, Y.-E. Sun, and K.-J. Kim, Phys. Rev. ST Accel. Beams 11, 080701 (2008).

[6] H. L. Andrews, C. A. Brau, J. D. Jarvis, C. F. Guertin, A. O'Donnell, B. Durant, T. H. Lowell, and M. R. Mross, in Proceedings of the 30th International Free-Electron Laser Conference, Gyeongju, Korea, 2008, Paper No. MOPPH047.
[7] S. E. Korbly, A. S. Kesar, J. R. Sirigiri, and R. J. Temkin, Phys. Rev. Lett. 94, 054803 (2005).

[8] T. Ochiai and K. Ohtaka, Opt. Express 14, 7378 (2006).

[9] M. C. Lampel, Nucl. Instrum. Methods Phys. Res., Sect. A 385, 19 (1997).

[10] D. C. Nguyen, Nucl. Instrum. Methods Phys. Res., Sect. A 393, 514 (1997).

[11] S. E. Korbly, A. S. Kesar, R. J. Temkin, and J. H. Brownell, Phys. Rev. ST Accel. Beams 9, 022802 (2006).

[12] A. Doria, G. P. Gallerano, E. Giovenale, G. Messina, G. Doucas, M.F. Kimmitt, H.L. Andrews, and J.H. Brownell, Nucl. Instrum. Methods Phys. Res., Sect. A 483, 263 (2002).

[13] G. Doucas, M. F. Kimmitt, A. Doria, G. P. Gallerano, E. Giovenale, G. Messina, H.L. Andrews, and J.H. Brownell, Phys. Rev. ST Accel. Beams 5, 072802 (2002).

[14] G. Doucas, V. Blackmore, B. Ottewell, C. Perry, P. G. Huggard, E. Castro-Camus, M. B. Johnston, J. Lloyd Hughes, M.F. Kimmitt, B. Redlich, and A. van der Meer, Phys. Rev. ST Accel. Beams 9, 092801 (2006).

[15] V. Blackmore, G. Doucas, C. Perry, B. Ottewell, M. F. Kimmitt, M. Woods, S. Molloy, and R. Arnold, Phys. Rev. ST Accel. Beams 12, 032803 (2009).

[16] P. M. van den Berg, J. Opt. Soc. Am. 63, 689 (1973).

[17] P. M. van den Berg, J. Opt. Soc. Am. 63, 1588 (1973).

[18] O. Haeberlé, P. Rullhusen, J.-M. Salomé, and N. Maene, Phys. Rev. E 49, 3340 (1994).

[19] G. Kube, Nucl. Instrum. Methods Phys. Res., Sect. B 227, 180 (2005).

[20] J. H. Brownell, J. Walsh, and G. Doucas, Phys. Rev. E 57, 1075 (1998).

[21] S. R. Trotz, J. H. Brownell, J.E. Walsh, and G. Doucas, Phys. Rev. E 61, 7057 (2000).

[22] G. Kube et al., Phys. Rev. E 65, 056501 (2002).

[23] J. H. Brownell, G. Doucas, M. F. Kimmitt, J. H. Mulvey, M. Omori, and J. E. Walsh, J. Phys. D 30, 2478 (1997).

[24] D. V. Karlovets and A. P. Potylitsyn, Phys. Rev. ST Accel. Beams 9, 080701 (2006).

[25] J. T. Donohue and J. Gardelle, Phys. Rev. ST Accel. Beams 9, 060701 (2006).

[26] D. Li, K. Imasaki, Z. Yang, Gun-Sik Park, S. Miyamoto, S. Amano, and T. Mochizuki, Jpn. J. Appl. Phys. 46, 601 (2007).

[27] J. Gardelle and J. T. Donohue, Nucl. Instrum. Methods Phys. Res., Sect. B 266, 3822 (2008).

[28] D. Li, K. Imasaki, X. Gao, Z. Yang, and Gun-Sik Park, Appl. Phys. Lett. 91, 221506 (2007).

[29] A. S. Kesar, M. Hess, S. E. Korbly, and R. J. Temkin, Phys. Rev. E 71, 016501 (2005).

[30] A. S. Kesar, Phys. Rev. ST Accel. Beams 8, 072801 (2005).

[31] C. A. Balanis, Advanced Engineering Electromagnetics (John Wiley \& Sons, Inc., New York, 1989).

[32] A. F. Peterson, S. L. Ray, and R. Mittra, Computational Methods for Electromagnetics, IEEE/OUP Series on Electromagnetic Wave Theory (IEEE Press, New York, 1997).

[33] B. E. Barrowes, F. L. Teixeira, and J. A. Kong, Microw. Opt. Technol. Lett. 31, 28 (2001).

[34] G. Doucas (private communication). 